\title{
CFD Prediction of Heterogeneities in the Scale up of Liquid-Liquid Dispersions
}

\author{
R. Gelves and L. Niño
}

\begin{abstract}
In this work simulations were developed in order to allow the examinations of heterogeneities in the scale up of liquid-liquid dispersions (oil-water) in 3 and $300 \mathrm{~L}$ stirred tank bioreactors using CFD simulations (Computational Fluid Dynamics). The effects of turbulence, rotating flow, drop breakage were simulated by using the k-e, MRF (Multiple Reference Frame) and PBM (Population Balance Model), respectively, providing detailed information of important bioreactor conditions. CFD modeling predicts with good approach hydrodynamic trends which can affect the liquid-liquid dispersion process of large scale bioreactors. Motivated by these results, CFD simulations are qualified as a very promising tool for predicting hydrodynamics and drop sizes especially useful for liquid-liquid applications which are characterized by the challenging problem of emulsion stability due to undesired drop and heterogeneities in the scale up process.
\end{abstract}

Index Terms-Computational fluid dynamics, drop size, population balance model, bioreactor, scale up.

\section{INTRODUCTION}

The liquid-liquid dispersions in turbulent flow are common in many applications, including chemical, petroleum, pharmaceutical and food industries [1]. In these bioprocesses phase dispersion and drop size have a significant importance on emulsion stability. Hence, the bioreactor operational conditions is of great importance for producing acceptable drop size to ensure stability, especially when emulsion processes are planned in large scale [2]. The loss of complete mixing conditions with increasing scale could generate gradients leading to a departure from optimal conditions found in laboratory scale. Sharp gradients of liquid-liquid dispersions are caused by poor mixing generated by empirical methods adopting as scale up strategies. All empirical scale up criteria are based on ideal approximations to maintain constant an operating parameter, leaving aside the hydrodynamics and transport phenomena governing the scale up process. Knowing the hydrodynamic behavior on the scale up bioreactors allows identifying the degree of departure from perfect mixing conditions associated with scaling rules.

Drop breakage and coalescence during high-shear preparation have a substantial impact on the drop size distribution. While drop breakage conditions have been extensively studied, predictive models for hydrodynamics, drop breakage and coalescence under turbulent conditions are lacking in a scale up process [3]. Due the lack of suitable

Manscript received September 1, 2013; revised November 3, 2013

The authors are with the Universidad de Antioquia, Calle $67 \mathrm{~N}^{\circ} .53-108$ Medellín, Colombia (e-mail: egrg832@udea.edu.co, lilibethniolopez@ hotmail.com). models, emulsified products in the scale up liquid-liquid dispersions are currently developed by combining a broad knowledge of the previous product formulations with empirical scientific experimentation. Because this approach is intuitive and experimental, the progression of a formulation is generally unpredictable and a new product will often go through hundreds of prototype formulations in a laboratory or pilot plant before commercialization [3]. Due to the very large number of possible formulation and processing combinations that need to be explored in a scale up process, the traditional trial-and-error approach requires significant time and resources [3]. Hence it is the motivation of this work to present a CFD simulation approach for the estimation of drop size and heterogeneities due to the scale up process in liquid-liquid systems.

\section{METHODS}

A $3 \mathrm{~L}$ stirrer tank with $0.160 \mathrm{~m}$ diameter equipped with a 0,059 $\mathrm{m}$ Rushton turbine diameter and two cylindrical baffles were used for the experiments at lab scale and different stirrer speeds $(350,500$ and $650 \mathrm{rpm})$ were tested for defining the best condition [2] to implement in further scale up. It was used sunflower oil as dispersed phase. It was mainly used at concentration of $10 \%(\mathrm{v} / \mathrm{v})$ in de-ionized water at atmospheric pressure and a constant temperature of $20{ }^{\circ} \mathrm{C}$. Coalescence was reduced by sodium dodecyl sulfate - SDS provided by Sygma-Aldrich Co. An endoscope technique was developed [4], [5]. Considering this technique, drop size distributions for all phase fractions even under transient conditions [6], [7] can be determined with high time resolution.

The drops are semi-automatically measured and counted. The technique is capable of measuring drops of $25 \mu \mathrm{m}$ to $1000 \mu \mathrm{m}$ at any dispersed phase fraction. This technique offers reliable in-situ measurement of drop sizes from any part of the stirred vessel [5]. The endoscope technique (developed by Maass [5]) was applied in order to measure the drop size distributions as a function of parameters like stirrer speed.

Besides the general technical importance of analyzing the influence of these parameters, this set-up is very instructive for the analysis of the physical phenomena in stirred dispersions in a technically relevant parameter range. This is a major requirement for the development of CFD models for immiscible liquids dispersions [5].

For simulating heterogeneities in the scale up stage, a 300 $\mathrm{L}$ virtual bioreactor was dimensioned $(0.57 \mathrm{~m}$ diameter $)$ maintaining a geometric similarity from the tested $3 \mathrm{~L}$ bioreactor. Operational conditions were defined based on the best condition found in lab scale bioreactor (3 L) and using 
the $\mathrm{P} / \mathrm{V}$ (constant power input per liquid volume) empirical scale up criterion [8].

The Eulerian model is the most complex multiphase model in ANSYS FLUENT 13.0. It solves a system of $n$-momentum and continuity equations for each phase. The coupling is achieved through pressure and interfacial exchange coefficients. The mass conservation equation for each phase is shown below:

$$
\frac{\partial}{\partial t}\left(\rho_{i} \alpha_{i}\right)+\nabla \cdot\left(\alpha_{i} \rho_{i} \vec{U}_{i}\right)
$$

where $\rho_{i}, \alpha_{i}$ and $\vec{U}_{i}$ represent the density, volume fraction and the rate of phase $i$ (oil or water). It is assumed that the water phase and the oil phase share space in proportion to their volume, such that their volume fractions sums to unity in the cell domain.

$$
\propto_{G}+\propto_{L}=1.0
$$

The momentum equation for phase $i$ is described below:

$$
\begin{aligned}
& \frac{\partial}{\partial t}\left(\rho_{i} \alpha_{i} \vec{U}_{i}\right)+\nabla \cdot\left(\alpha_{i} \rho_{i} \vec{U}_{i} \vec{U}_{i}\right)=\alpha_{i} \nabla p \\
& \quad+\nabla \cdot \overline{\bar{\tau}}_{e f f i}+\vec{R}_{i}+\vec{F}_{i}+\alpha_{i} \rho_{i} \vec{g}
\end{aligned}
$$

$p$ is the pressure shared by both phases and $\vec{R}_{i}$ represents the interfacial momentum exchange. The $\vec{F}_{i}$ term represents the Coriolis and centrifugal forces expressed in the MRF model for rotating flows and is represented as:

$$
\vec{F}_{i}=-2 \alpha_{i} \rho_{i} \vec{N} \times \vec{U}_{i}-\alpha_{i} \rho_{i} \vec{N} \times(\vec{N} \times \vec{r})
$$

$\vec{N}$ is the angular velocity, $\vec{r}$ is the position vector. The Reynolds stress tensor $\overline{\bar{\tau}}_{e f f i}$ is related to the mean velocity gradients through the Boussinesq hypothesis [9]:

$$
\begin{gathered}
\overline{\bar{\tau}}_{e f f i}=\alpha_{i}\left(\mu_{\text {lam }, i}+\mu_{t, i}\right)\left(\nabla \vec{U}_{i}+\nabla \vec{U}_{i}^{T}\right) \\
-\frac{2}{3} \alpha_{i}\left(\rho_{i} k_{i}+\left(\mu_{\text {lam }}, i+\mu_{t, i}\right) \nabla \cdot \vec{U}_{i}\right) \overline{\bar{I}}
\end{gathered}
$$

$\mu_{\text {lam }, i}$ is the molecular viscosity of phase $i, \overline{\bar{I}}$, is the strain tensor.

The most important force exchange between the phases is drag force acting on drops. This force depends on friction, pressure, cohesion, and other hydrodynamic effects [10].

$$
R_{L}=-R_{G}=K\left(\vec{U}_{G}-\vec{U}_{L}\right)
$$

$K$ is the exchange coefficient of water and oil phases and is determined by the equation:

$$
K=\frac{3}{4} \rho_{L} \alpha_{L} \alpha_{G} \frac{C_{D}}{d}\left|\vec{U}_{G}-\vec{U}_{L}\right|
$$

$d$ is the drop diameter and the drag coefficient and $C_{D}$ is defined as a function of Reynolds number:

$$
R e_{p}=\frac{\rho_{L}\left|\vec{U}_{G}-\vec{U}_{L}\right| d}{\mu_{L}}
$$

To calculate the drag coefficient using the standard correlation [11]:

$$
C_{D}=\left\{\begin{array}{ll}
\frac{24\left(1+0.15 R e_{p}^{0.687}\right)}{R e_{p}}, & R e_{p} \leq 1000 \\
0.44, & R e_{p}>1000
\end{array}\right\}
$$

The dispersed turbulence $k-\varepsilon$ model can be considered as the multiphase standard turbulence model. It represents the extension of the single phase $k-\varepsilon$ model and is used when the secondary phase concentrations are diluted on primary phase. $k$ and $\varepsilon$ equations describing this model are as follows:

$$
\begin{gathered}
\frac{\partial}{\partial t}\left(\rho_{L} \alpha_{L} k_{L}\right)+\nabla \cdot\left(\rho_{L} \alpha_{L} \vec{U}_{L} k_{L}\right) \\
=\nabla \cdot\left(\alpha_{L} \frac{\mu_{t, L} \nabla k_{L}}{\sigma k}\right)+\alpha_{L} G_{k, L}-\alpha_{L} \rho_{L} \varepsilon_{L} \\
+\alpha_{L} \rho_{L} \Pi_{K, L}
\end{gathered}
$$

$$
\begin{gathered}
\frac{\partial}{\partial t}\left(\rho_{L} \alpha_{L} k_{L} \varepsilon_{L}\right)+\nabla \cdot\left(\rho_{L} \alpha_{L} \vec{U}_{L} \varepsilon_{L}\right) \\
=\nabla \cdot\left(\alpha_{L} \frac{\mu_{t, L} \nabla \varepsilon_{L}}{\sigma \varepsilon}\right)+\alpha_{L} \frac{\varepsilon_{L}}{k_{L}}\left(C_{1 \varepsilon} G_{k, L}-C_{2 \varepsilon} \rho_{L} \varepsilon_{L}\right) \\
+\alpha_{L} \rho_{L} \prod_{\varepsilon, L}
\end{gathered}
$$

In these equations, $G_{k, L}$ represents the generation of turbulent kinetic energy $k_{L}$ of the water phase due to mean velocity gradients, $\varepsilon_{L}$ is the turbulent dissipation energy. $\Pi_{K, L}$ and $\Pi_{\varepsilon, L}$ represent the influence of the dispersed oil phase in the continuous phase and are modeled with the Elgobashi and Rizk [7] equations.

The turbulent viscosity $\mu_{t, L}$, is calculated from:

$$
\mu_{t, L}=\rho_{L} C_{\mu} \frac{k_{L}^{2}}{\varepsilon_{L}}
$$

The values of the constants used in this experiment were $C_{1 \varepsilon}: 1.44, C_{2 \varepsilon}: 1.92, C_{\mu}: 0.09 \sigma_{k}: 1.00$ and $\sigma_{\varepsilon}: 1.30 . \sigma_{k}$ and $\sigma_{\varepsilon}$ represent turbulent Prandtl number for $k$ and $\varepsilon$, respectively.

The eulerian approach was coupled to population balance models. The finite volume technique was used in Ansys Fluent 13 to solve the equations. The effects of turbulence and rotating flow were simulated by using the k-e and MRF (Multiple Reference Frame) models. The discrete method [12] is used in this manuscript to solve the population balance equations. The drop population is discretized into a finite number of intervals of drop sizes. The population balance equations for different drop classes can be written as [13]:

$$
\frac{\partial}{\partial t}\left(\rho_{G} n_{i}\right)+\nabla \cdot\left(\rho_{G} \vec{U}_{G} n_{i}\right)=\rho_{G}\left(\Gamma_{B_{i_{B}}}-\Gamma_{D_{i_{B}}}\right)
$$

where $n_{i}$ is the number of classes of drop $i, \Gamma_{B_{i_{B}}}$ is the birth rate due to breakage and $\Gamma_{D_{i_{B}}}$ is the death rate. The terms of breakage are:

$$
\Gamma_{B_{i_{B}}}=\int_{\Omega_{v}} p g\left(v^{\prime}\right) \beta\left(v \mid v^{\prime}\right) n\left(v^{\prime}, t\right) d v^{\prime}
$$




$$
\Gamma_{D_{i_{B}}}=g(v) n(v, t)
$$

$g(v)$ is the breakup rate of drops of size $v ; g\left(v^{\prime}\right)$, is the breakup frequency of drop $v^{\prime}$ and $\beta\left(v \mid v^{\prime}\right)$ is the probability density function of drops broken from the volume $v^{\prime}$ in a drop of volume $v$. The drop breakup is analyzed in terms of drops interaction with turbulent eddies. These turbulent eddies increase the drop surface energy to cause deformation. The breakup occurs if the increase in the surface energy reaches a critical value. The breakup rate is defined as [14]:

$$
\begin{gathered}
g\left(v^{\prime}\right) \beta\left(v \mid v^{\prime}\right)=k \int_{\xi_{\min }}^{1} \frac{(1+\xi)^{2}}{\xi^{11 / 3}} \exp \left(-b \xi^{-11 / 3}\right) d \xi \\
k=0.9238 \varepsilon^{1 / 3} d^{-2 / 3} \alpha \\
b=12\left(f^{2 / 3}+(1-f)^{2 / 3}-1\right) \sigma \rho^{-1} \varepsilon^{-2 / 3} d^{-5 / 3}
\end{gathered}
$$

where $d$ is the particle diameter, $\xi$ is the dimensionless eddy size, $f$ is the breakage frequency.

The 3D mesh is composed for hybrid cells with 25000 and 450000 computational cells for 3 and $300 \mathrm{~L}$ bioreactors, respectively (Fig. 1 and Fig. 2). The finite volume technique implemented in the CFD code Ansys Fluent 13.0 Software was used to convert the Navier- Stokes equations into algebraic equations which can be solved numerically. Tank walls, stirrer surfaces and baffles are treated with no slip conditions and standard wall functions.

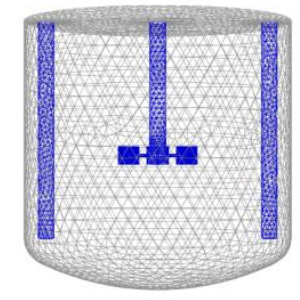

Fig. 1. Mesh generation for the liquid-liquid system (3 L bioreactor).

To solve the partial differential equations the PC SIMPLE algorithm was used which couples pressure and velocity. The second order Upwind scheme was applied for the spatial terms. It was assumed that the solution converges when the scaled residuals remain with values smaller than $10^{-5}$ and when the pseudo-regime for sauter diameter is reached.

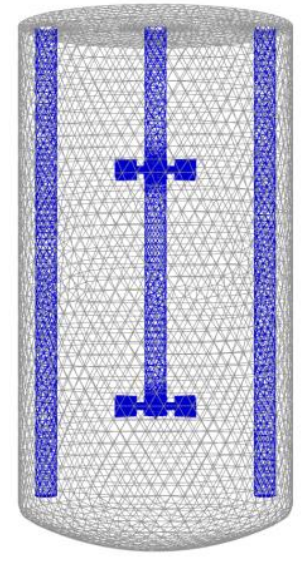

Fig. 2. Mesh generation for the liquid-liquid system (300 L bioreactor).

\section{RESUlts}

The mean goal of this work was to study the heterogeneities due to loss of complete mixing conditions with increasing scale, using CFD simulations. Special emphasis was given to the elucidation of liquid-liquid volume fraction dispersion and drop size distributions. Operational conditions were defined based on the best condition found in the $3 \mathrm{~L}$ lab scale bioreactor [2] and using the $\mathrm{P} / \mathrm{V}$ (constant power input per liquid volume) empirical scale up criterion. Fig. 3 and Fig. 4 show the oil volume fractions for the liquid-liquid system in 3 and $300 \mathrm{~L}$ bioreactor simulated by CFD.

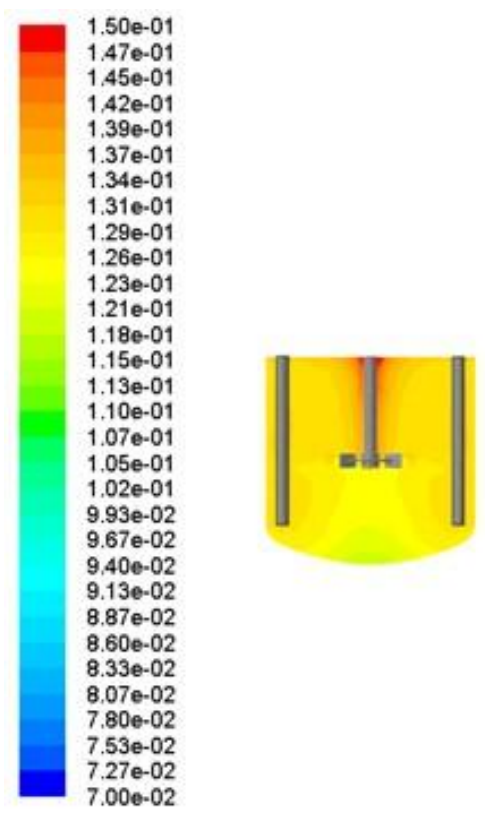

Fig. 3. Oil volume fractions [-] for the liquid-liquid system (3 L bioreactor).

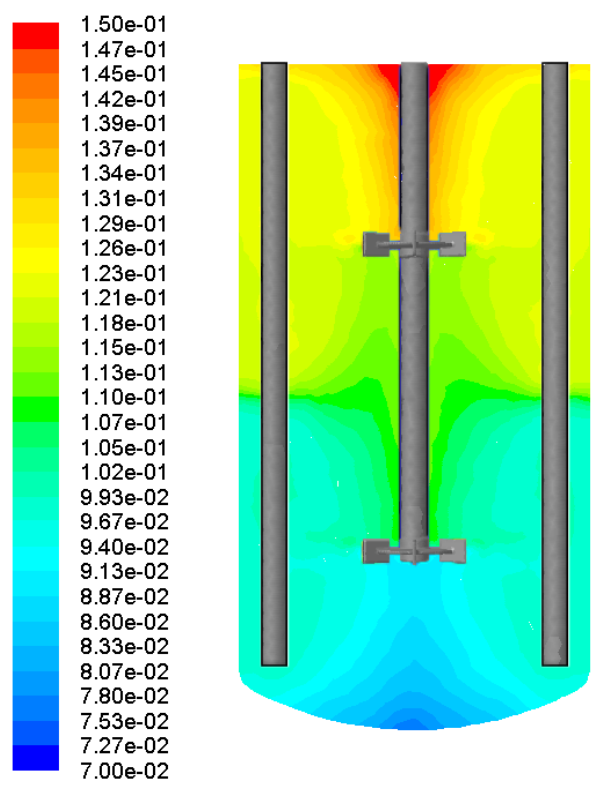

Fig. 4. Oil volume fractions [-] for the liquid-liquid system (300 L bioreactor).

Simulating the scale up in $300 \mathrm{~L}$, it can be seen that relatively low oil dispersion occurs especially in bottom of bioreactor because of low centrifugal forces generated by the Rushton turbine. Obviously, centrifugal forces and turbulence dissipation energies could not overcome the oil 
immiscibility in water and the appearance of heterogeneous environment is becoming important for the liquid-liquid dispersion because emulsion stability can be affected. Contrarily, oil gradients are minimal when the stirrer tank is operated at lab scale $(3 \mathrm{~L})$ due to increase in turbulence dissipation energy [2].

Moreover, Fig. 5 and Fig. 6 depict another reason for explaining the high discrepancy between 3 and $300 \mathrm{~L}$ oil vol ume fraction dispersion. Turbulence dissipation energy profiles in both scales are significantly different.

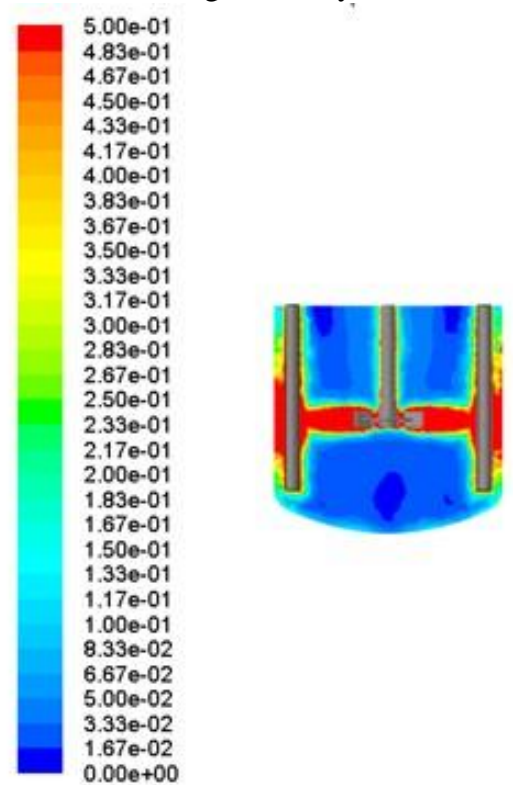

Fig. 5. Turbulence dissipation energy $\left[\mathrm{m}^{2} / \mathrm{s}^{3}\right]$ for the liquid-liquid system (3 L bioreactor) [17].
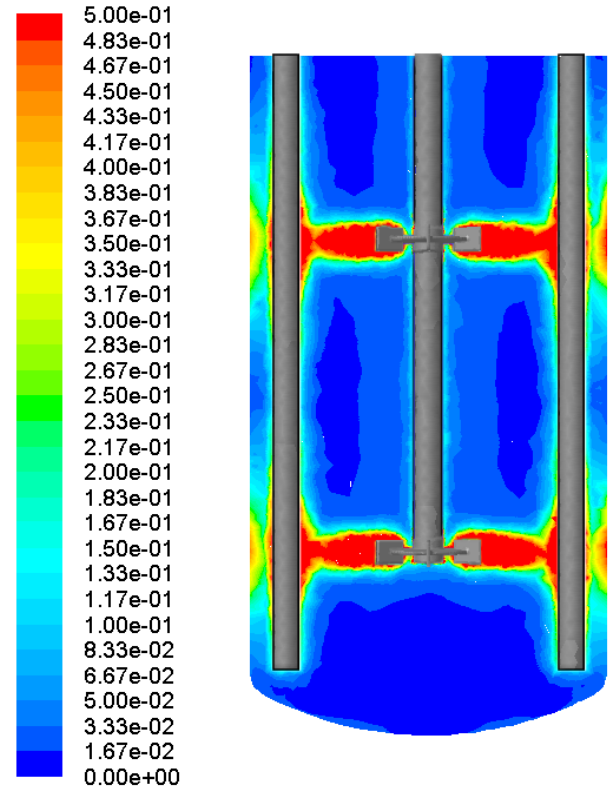

Fig. 6. Turbulence dissipation energy $\left[\mathrm{m}^{2} / \mathrm{s}^{3}\right]$ for the liquid-liquid system (300 L bioreactor).

While the lab scale stirred tank bioreactor shows well-defined turbulence dissipation energy zones between the impeller discharge region, walls and baffles, the $300 \mathrm{~L}$ bioreactor shows dead zones on bottom bioreactor. Consequently, poor mixed lower region of the bioreactor are more significant due to loss of complete mixing conditions with increasing scale.
Fig. 7 and Fig. 8 show the velocity magnitude calculated for the lab scale stirred tank and $300 \mathrm{~L}$ bioreactors. It is observed that highest velocities are found in the region closed to the rotating Rushton turbines. Moreover, the mean velocity decreases gradually away from the Impeller and becomes very low at the bottom of the bioreactors especially in $300 \mathrm{~L}$ scale. Noteworthy, similar results were found by Gelves [10] who analyzed CFD simulations of a Rushton turbine.

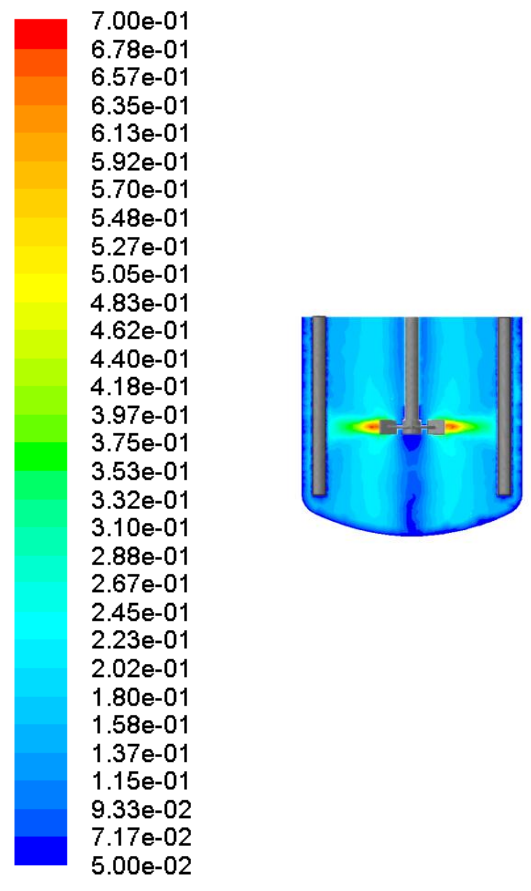

Fig. 7. Oil velocity magnitude $[\mathrm{m} / \mathrm{s}]$ for the liquid-liquid system $(3 \mathrm{~L}$ bioreactor) [17].
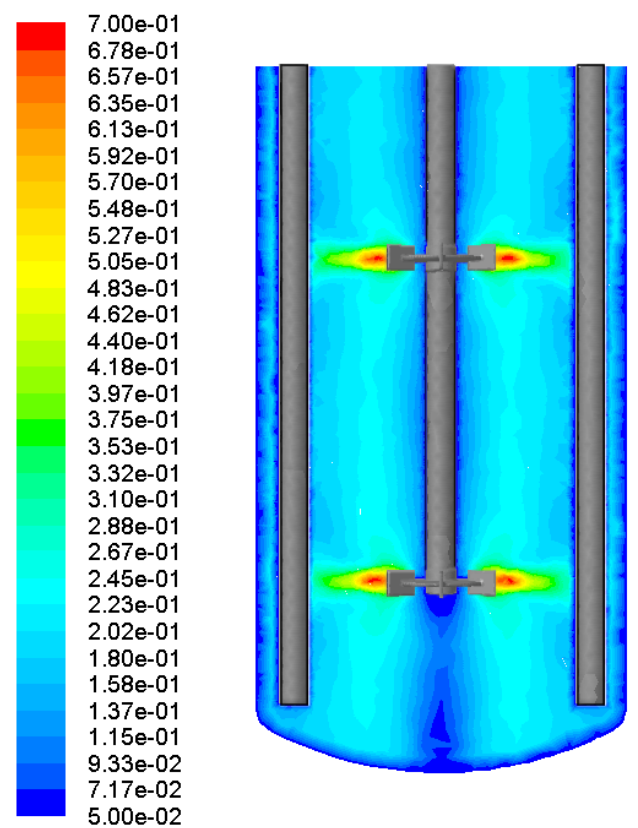

Fig. 8. Oil velocity magnitude [m/s] for the liquid-liquid system $(300 \mathrm{~L}$ bioreactor).

The different liquid velocity contours are also checked by the analysis of Kolmogorov length scale $\lambda_{K}[15]$ :

$$
\lambda_{K}=\left(\frac{\varepsilon_{L}}{v^{3}}\right)^{-\frac{1}{4}}
$$


with $\varepsilon_{L}$ being the turbulence dissipation energy calculated from CFD and $v$ the dynamic viscosity of the liquid. Following this definition, the Kolmogorov diameter investigates eddies, created at the "hot spots" of energy input [10]. According to the hypothesis, only eddies with length scale smaller than drop diameters can induce breakage. Larger eddies lead to drop transport instead [14]. Fig. 9 and Fig. 10 show the Kolmogorov length scale $\lambda_{K}$ simulated by CFD in both scales ( 3 and $300 \mathrm{~L}$ ). Considering these results, smaller length scales, which dominate high breakage phenomenon, are present in lab scale bioreactor than $300 \mathrm{~L}$ bioreactor. This finding can also explain the heterogeneities found at $300 \mathrm{~L}$ scale.

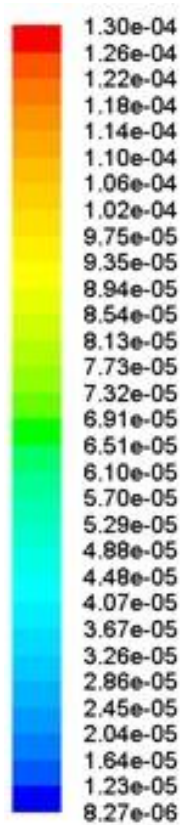

Fig. 9. Kolmogorov length scale [m] for the liquid-liquid system $(3 \mathrm{~L}$ bioreactor).

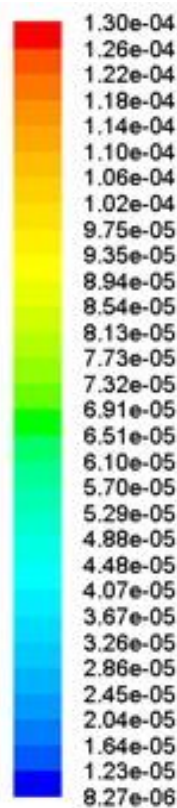

Fig. 10. Kolmogorov length scale [m] for the liquid-liquid system $(300 \mathrm{~L}$ bioreactor).

In these bioprocesses phase dispersion and drop size have a significant importance on emulsion stability. Hence, the bioreactor operational conditions are of great importance for producing acceptable drop size to ensure stability, especially when emulsion processes are planned in large scale. The most relevant parameter for analyzing these hydrodynamic mechanisms is the sauter diameter. Fig. 11 and Fig. 12 show the sauter mean diameter distribution simulated using CFD in both scales (3 and 300L).

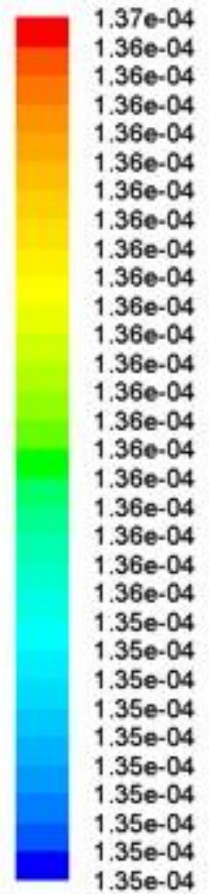

Fig. 11. Sauter mean diameter [m] for the liquid-liquid system (3 L bioreactor).

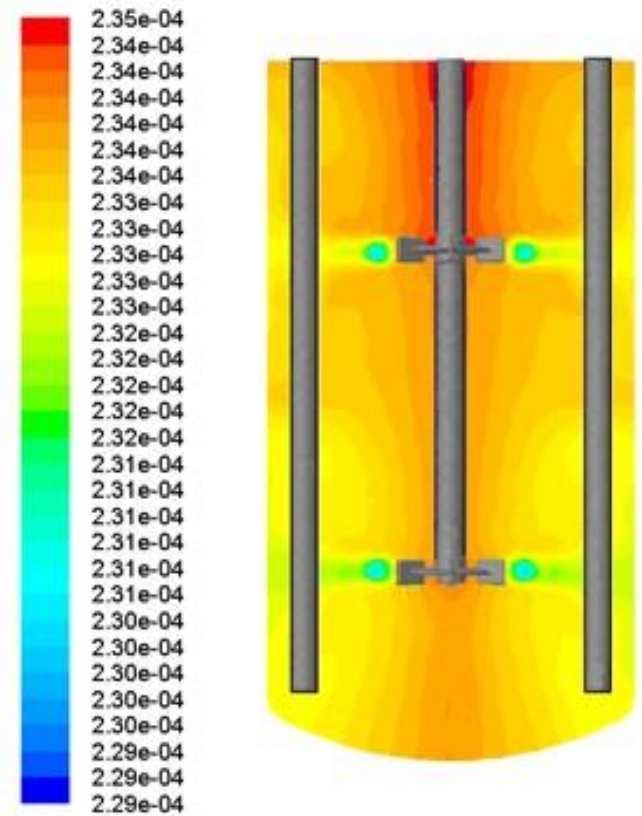

Fig. 12. Sauter mean diameter [m] for the liquid-liquid system $(300 \mathrm{~L}$ bioreactor).

It can be interpreted as a parameter, summarizing all individual impacts affecting the drop sizes and their distributions in one single value [10]. It is estimated coupling CFD-based hydrodynamics with population balance models (see equation (13)). Interestingly, lowest values of $\sim 136 \mu \mathrm{m}$ were found close to the blades in the cross-sectional areas of the agitator speed direction. These regions mirror relatively high, local drop breakage rates.

In the case of $300 \mathrm{~L}$ bioreactor (Fig. 12) the sauter diameter 
was higher $(229-235 \mu \mathrm{m})$ than the values found for $3 \mathrm{~L}$ bioreactor (Fig. 11). Besides, the 300L bioreactor reveals a significantly non-homogeneous sauter mean diameter distribution, while special differences are minimal using the 3 $\mathrm{L}$ bioreactor. The latter is the consequence of the improved hydrodynamics which promotes the formation of uniform bubble sizes, generally caused by the mixing.

Moreover, experimental sauter diameter was performed at $3 \mathrm{~L}$ scales [2] using an endoscope prove and compared to CFD results (Table I).

TABLE I: CFD AND EXPERIMENTAL RESULTS (3 L)

\begin{tabular}{ccccccc}
\multicolumn{8}{c}{ TABLE I: CFD AND EXPERIMENTAL RESULTS (3 L) } \\
\hline \hline $\mathrm{rpm}$ & \multicolumn{3}{c}{350} & \multicolumn{2}{c}{500} & \multicolumn{2}{c}{650} \\
\hline $\mathrm{d}_{32}$ & CFD & Exp & CFD & Exp & CFD & Exp \\
{$[\mu \mathrm{m}]$} & 248 & 242 & 145 & 151 & 136 & 122 \\
\hline \hline
\end{tabular}

Interestingly, CFD simulations show that lowest drop size values are reached at $650 \mathrm{rpm}(137 \mu \mathrm{m})$. Independent drop size measurements by an experimental endoscope technique applying the same operational conditions confirmed these findings [2]. For that reason $650 \mathrm{rpm}$ was chosen in this work at starting point for calculating the operational conditions at $300 \mathrm{~L}$ based on P/V constant. The very good agreement is qualified as an evidence for suitability of the breakage terms used in CFD to formulate the population balance models. Besides it provides the sound basis for the discussion of the oil volume contour plots and the resulting $d_{32}$ distributions. Both represent the backbone for the comparison of the emulsion stability not only for lab but also for large scale bioreactors.

\section{CONCLUSION}

The numerical results from a scale up process of liquid-liquid dispersions are analyzed using CFD. Possible heterogeneities were simulated at $300 \mathrm{~L}$ bioreactor due to poor mixing conditions reached at this scale. Motivated by these simulated and experimental results CFD simulations are qualified as a very promising tool for predicting hydrodynamics and drop sizes especially useful for liquid-liquid applications which are characterized by the challenging problem of emulsion stability due to undesired drop sizes in large scale bioprocess.

\section{ACKNOWLEDGMENT}

The authors gratefully acknowledge DAAD and Universität Berlin for their financial support of this internship.

\section{REFERENCES}

[1] W. Podgórska, "Influence of the impeller type on drop size in liquid-liquid dispersions," presented at the 13th European Conference on Mixing London, 2009.

[2] R. Gelves, "CFD Simulations of liquid-liquid dispersions in a stirred tank bioreactor," presented at the ICNAAM, Rhodes, Greece,
September 21-27, 2013. Reprinted with permission. Copyright 2013. AIP Publishing LLC.

[3] B. Raikar, R. Bhatia, F. Malone, and A. Henson, "Experimental studies and population balance equation models for breakage prediction of emulsion drop size distributions," Chemical Engineering Science, vol. 64, pp. 2433-2447, 2009.

[4] J. Ritter and M. Kraume, "On-line measurement technique for drop size distributions in liquid/liquid systems at high dispersed phase fractions," Chemical Engineering and Technology, vol. 23, no. 7, pp. $579-582,2000$.

[5] S. Maaß, S. Wollny, J. Rojahn, A. R. Paschedag, and M. Kraume, "Comparison of measurement techniques for drop sizes in highly dispersed and fastly coalescing systems," PARTEC, Nuremberg, pp. 27-29, 2007

[6] M. Wegener, S. Schlauch, and M. Kraume, "Transiente tropfengrößenverteilungen in gerührten Flüssig/flüssig-dispersionen," Chemie Ingenieur Technik, vol. 77, no. 1-2, pp. 80-84, 2004.

[7] M. Wegener, A. R. Paschedag, and M. Kraume, "The effect of $\mathrm{pH}$ on experimental and simulation results of transient drop size distributions in stirred liquid-liquid dispersions," Chemical Engineering Science, vol. 61, no. 9, pp. 3018-3024, 2004.

[8] A. Junker, "Scale-up methodologies for escherichia coli and yeast fermentation processes," Journal of Bioscience and Bioengineering, vol. 97, pp. 347-364, 2004.

[9] G. R. Kasat, A. B. Pandit, and V. V. Ranade, "CFD simulation of gas-liquid flows in a reactor stirred by dual rushton turbines," International Journal of Chemical Reactor Engineering, vol. 6, pp. $1-28,2008$.

[10] R. Gelves, A. Dietrich, and R. Takors, "Modeling of gas-liquid mass transfer in a stirred tank bioreactor agitated by a rushton turbine or a new pitched blade impeller," Bioprocess and Biosystem Engineering, pp. 1-2, Jul. 5, 2013.

[11] M. Ishii and N. Zuber, "Drag coefficient and relative velocity in bubbly, droplet or particulate flows," AIChE Journal, vol. 25, pp. 843-855, 1979.

[12] S. E. Elgobashi and M. A. Rizk, "A two-equation turbulence model for dispersed dilute confined two-phase flows," Int. J. of Multiph Flow, vol. 15, pp. 119-133, 1998

[13] P. Chen, M. P. Dudukovic, and J. Sanyal, "Numerical simulation of bubble columns flows: Effect of different breakup and coalescence closures," Chem Eng Sci, vol. 60, pp. 1085-1101, 2005.

[14] J. Y. Luo, R. I. Issa, and A. D. Gosman, "Prediction of impeller induced flows in mixing vessels using multiple frames of reference," IchemE Symposium Series, Institution of Chemical Engineers, vol. 136, pp. 549-556, 1994.

[15] A. W. Nienow, "Reactor engineering in large animal cell culture," Cytotechnology, vol. 50, pp. 9-33, 2006.

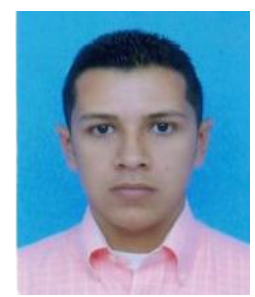

R. Gelves was born in Cucuta, Colombia, on May 10, 1981. He received his bachelor's degree in Biotechnology Engineering from Universidad Fancisco de Paula Santander, Colombia. In 2010, he won a scholarship from DAAD for working with Endoscopy Techniques at Universität Berlin, Germany. Then, he worked at Universität Stuttgart, Germany by performing CFD simulations (Computational Fluid Dynamics) of a Scale Up Bioprocess for Animal Cell Cultures. Actually, he is about to finish his PhD at the Universidad de Antioquia, Colombia. His area of interest is the Computational Fluid Dynamics (CFD) applied to Biochemical Engineering.

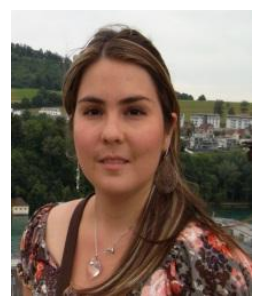

L. Niño was born in Cucuta, Colombia, on December 28, 1984. She received her bachelor's degree in Biotechnology Engineering from Universidad Fancisco de Paula Santander, Colombia. In February 2013, she finished her master's degree in Biology from Universidad e Antioquia. Her area of interest is the Scale Up of Plat Cell Cultures. 